\title{
Prevalence of Extended Spectrum Beta Lactamase (ESBL) Producing Gram Negative Bacilli from various clinical isolates.
}

\author{
Dr Nazneen Siddiqui, Dr Jayshree Bhakre, Dr Ajit Damle, Dr Jyoti Bajaj \\ (Department Of Microbiology, Govt Medical College and Hospital, Aurangabad, Maharashtra, India)
}

\begin{abstract}
Rapid emergence of ESBL production in GNB has put forward a major challenge in their detection as well as treatment options. Therefore for proper management of these resistant bacteria it is very important and necessary to correctly identify them. Aim: To know the prevalence of ESBL producing GNBS in all clinical isolates received in the Microbiology Laboratory of GMCH Aurangabad. Method: The study was conducted from June 2008 to July 2009. Our study group included 711 GNB,s identified by Grams staining, colony morphology and biochemical reactions. AST was done on MHA by Kirby Beurs disc diffusion method. All the organisms suspected of producing ESBL by the CLSI screening criteria were confirmed by the phenotypic confirmatory disc diffusion test (PCDDT). Result: Out of the 711 GNB tested, 433(61\%) were found to be ESBL positive by the PCDDT method. The different GNB's identified were Klebsiella pneumoniae (47\%) E. coli (23\%), Pseudomonas spp. (21.37\%), etc. ESBL production was most common in Klebsiella pneumoniae (74\%), followed by E. coli (62\%), Enterobacter spp. (60\%) etc. Imepenem showed the highest rate of sensitivity (97\%). Conclusion: Since ESBL producing GNBs are on a constant rise, it is very necessary to test all the organisms for ESBL production by the screening and confirmatory methods. Spread of these ESBL producers should be prevented by infection control policy and empirical use of antibiotics.
\end{abstract}

Keywords: ESBL, Infection control policy, Multidrug resistance, PCDDT, Prevalence,

\section{Introduction}

The fear of antibiotic resistance has gripped the whole world like a wild fire. Man is working hard for taking out ways to fight these organisms but in turn these organisms are working much harder for finding ways to counter his each and every move. . We human beings, the so called superior beings, are under the threat of the minutest structures on this earth. Is it that these organisms are better versed with the theory of "SURVIVAL OF THE FITTEST" then we are???

Antibiotic resistance among Gram-negative bacilli (GNB) is a rapidly expanding problem due to the organisms' ability to mutate, and to acquire and transmit plasmids and other mobile genetic elements encoding resistance genes. ${ }^{[1]}$ Beta-lactamase antibiotics are among the most widely prescribed antibiotics worldwide. and The emergence of resistance to these agents has resulted in a major clinical crisis. ${ }^{[2]}$

The introduction of the third-generation cephalosporins into clinical practice in the early 1980s was heralded as a major breakthrough in the fight against $\beta$-lactamase-mediated bacterial resistance to antibiotics. The first report of plasmid-encoded B-lactamases capable of hydrolyzing the extended-spectrum cephalosporins was published in $1983 .{ }^{[3]}$ Mutations within the structural genes encoding the older enzymes had occurred, giving rise to derivatives that possessed an extended substrate profile compared with that of the parental enzymes. Thus, these new enzymes were given the name Extended-spectrum beta lactamases (ESBLs) to reflect the fact that they were derivatives of older enzymes and had a new capability to hydrolyze a broader spectrum of $\beta$ lactam drugs. ${ }^{[4]}$ In 1983, the first reported Extended-spectrum beta-lactamase (ESBL) producer occurred in K. pneumoniae followed by the detection of an ESBL producer in E. coli in $1987 .{ }^{[5]}$ Since their initial description in Germany in 1983, ESBLs have diversified and spread worldwide.

ESBL producing strains are Klebsiella pneumoniae, Klebsiella oxytoca and Escherichia coli. ${ }^{[4]}$ Other organisms reported to harbour ESBLs include Enterobacter spp., Salmonella, Morganella morganii, Proteus spp., Serratia marcescens and Pseudomonas aeruginosa. However, the frequency of ESBL production in these organisms is low, ${ }^{[6]}$ but still they cannot be ignored.

Infections caused by ESBL-producers often exhibit a multidrug-resistant phenotype, leaving only a few reliable therapeutic options. So they are always associated with increased morbidity, mortality and health careassociated costs particularly in the Intensive-care settings. ${ }^{[7]}$

The common infections with ESBL-producing organisms include all the infections caused by gramnegative organisms such as urinary tract infections, peritonitis, cholangitis, intra-abdominal abscess, pneumonia, catheter-associated blood stream infections. ${ }^{[8]}$

There are indications that poor outcome occurred when patients with serious infections caused by ESBL producing organisms were treated with antibiotics to which the organisms were resistant. ${ }^{[9]}$ Since Enterobacteriaceae group are the most frequently isolated organisms from clinical specimens and considering 
the high prevalence of ESBL production in this group there is a great need for laboratory testing methods for correctly detecting these enzymes for proper management.

Focusing on this ever increasing issue of ESBL and considering the fact that no data was available on ESBL producing GNB's in our region, the current study was undertaken in our department just to add a few drops of knowledge into the vast ocean of ESBL.

\section{Material And Methods}

2.1Clinical isolates: The present study was conducted in the Department of Microbiology, GMC Aurangabad, extending over a period of 1 year from june 2008 to july 2009. A total of 2000 properly collected, well labelled samples, received in the Microbiology laboratory were processed following standard protocol ${ }^{[10]}$. $711 \mathrm{GNB}$ 's were isolated on the basis of Grams staining and were confirmed and identified to the species level by standard biochemical tests ${ }^{[10]}$. These were considered as our study group. The various samples which were received included pus, wound swabs, urine, blood, respiratory samples and other body fluids.

2.2 Antimicrobial susceptibility testing: The isolates were tested by the disc diffusion method (modified Kirby-Bauer method) on Muller Hinton agar (Hi-Media) using 0.5 McFarland's as the turbidity standard as per CLSI guidelines ${ }^{[11]}$. The sensitivity of the isolates to Third Generation Cephalosporins (3GC) viz., ceftazidime, cefotaxime, cefpodoxime, each $30 \mu \mathrm{g} / \mathrm{disc}$ and to the other antibiotics such as amikacin $(30 \mu \mathrm{g})$, gentamycin $(10$ $\mu \mathrm{g})$, ciprofloxacin $(5 \mu \mathrm{g})$, piperacillin $(30 \mu \mathrm{g})$, imipenem $(30 \mu \mathrm{g})$, (Hi-Media, India) was determined by the zone size criteria which was recommended by the $\mathrm{CLSI}^{[12]}$.

Quality control strains used for AST were:

- Escherichia coli ATCC 25922.

- Pseudomonas aeruginosa ATCC 27853.

- Klebsiella pneumonia ATCC 700603.

2.3 Detection Of ESBL: In the present study 711 Gram-negative bacilli were tested for ESBL production by CLSI ${ }^{[12]}$ recommended screening and confirmatory methods.

\subsection{CLSI ESBL Screening Test:}

As per the CLSI Screening criteria, the strain was considered to be "suspicious" of ESBL production if a zone diameter for the third generation cephalosporins was recorded as $<22 \mathrm{~mm}$ for ceftazidime, $<27 \mathrm{~mm}$ for cefotaxime and $<17 \mathrm{~mm}$ for cefpodoxime.

\subsection{CLSI ESBL Confirmatory Method:}

All the strains which were screened out for ESBL production were also subjected to confirmation by using the PCDDT, as recommended by the CLSI. ${ }^{[12]}$ Discs of ceftazidime + clavulanic acid and cefotaxim + clavulanic acid were used as per CLSI guidelines. ${ }^{[i 2]}$ Organism was considered as ESBL producer if there was a more than $5 \mathrm{~mm}$ increase in zone diameter for ceftazidime and cefotaxime tested in combination with clavulanic acid versus its zone when tested alone.

\section{Result}

The present study was conducted in the Department of Microbiology, Government Medical College, Aurangabad, Maharashtra, from June2008 to July 2009, to know the prevalence of ESBL producing gram negative bacilli in various clinical isolates at our tertiary health care centre.

Out of the total 2000 samples processed, 711 Gram-negative bacilli were isolated and further processed as our study group. Out of the 711 gram negative bacilli, 433(61\%) were confirmed as ESBL producers. Among the study group Klebsiella pneumoniae (47\%) was the most common organism isolated followed by E. coli.(23\%),Pseudomonas spp. 21.37\%, Acinetobacter spp. 2.3\%, Enterobacter spp. (2.1\%) and Proteus mirabilis (1.4\%) ESBL production was highest (74\%) in Klebsiella pneumonia, followed in frequency by E. coli( $62 \%)$, Enterobacter(60\%), Pseudomonas (38\%) etc.

Out of the total 711 isolates ,519 (73\%) turned out to be ESBL screen positive as per the CLSI screening criteria and among these 519 screen positive isolates , $433(83.42 \%)$ were confirmed as ESBL producers by CLSI confirmatory method. The specimen wise distribution of ESBL was as follows- urine (71\%), blood (68\%), pus $(57 \%)$,and other body fluids $(41 \%)$.Antibiotic resistance was significantly $(\mathrm{P}<0.001)$ more prevalent in ESBL positive isolates as compared to Non-ESBL isolates. Among all the ESBL producers highest sensitivity was recorded against Imepenem (97\%). and resistance generated by these ESBLs against other drugs like amikacin, ciprofloxacin and gentamicin was $46 \%, 59 \%$ and $63 \%$ respectively. 


\section{Discussion}

ESBLs in Gram negative bacteria have emerged as a major problem in hospitalized as well as community based patients. Nosocomial infections with Gram-negative bacilli are not uncommon in the local setting and can be perceived as a growing threat to public health. Since Gram-negative bacilli are the most frequently isolated organisms from clinical specimens and considering the high prevalence of ESBL production in this group there is a great need for laboratory testing methods for correctly detecting these enzymes for proper management. No doubt that Klebsiella and E. coli are most frequent GNBs causing disease as well as producing ESBLs, but the remaining GNBs can not be ignored. Though minor but they do cause severe diseases and hence should also be taken care of. Prevalence of different organisms in different hospital settings changes depending on the geographical area, type of sample, wards etc.In our study the distribution of GNBs was as follows:

Klebsiella pneumoniae $(47 \%)$ was the most common organism isolated. This was similar to other studies, conducted by Menon et al $(47.14 \%)^{[13]}$, Shobha et al $(45.62 \%)^{[9]}$, and Nevine et al $(41.17 \%)^{[7]}$.

The prevalence of ESBL among clinical isolates varies greatly worldwide and in geographic areas and is rapidly changing over time ${ }^{[14]}$. It varies from country to country and institution to institution. In our study the incidence of ESBL was found to be $61 \%$. In a study conducted by Mathur et al, in 2001, showed $68 \%$ isolates as ESBL producers ${ }^{[15]}$. Similarly a study from Malaysia in 2001 by Nurul et al depicted a prevalence of ESBL as $58.6 \%{ }^{[1]}$ Nevine et al in 2002 showed a prevalence of ESBL as $65.8 \%{ }^{[7]}$. Our results were similar with the above mentioned studies. But studies conducted by Ali et al $(45 \%)^{[2]}$, Rodrigues et al $(6.9 \%)^{[16]}$ and Menon et al $(20 \%)^{[13]}$, had results lower than ours. This wide variation could be explained by the high amount of geographical variation shown by ESBL producers. ESBLs are most commonly found in Klebsiella spp. and E. coli, but they have also been detected in other members of the Enterobacteriaceae family. Klebsiella is the genus which frequently harbours ESBL ${ }^{[17]}$ as was seen in our study. In our study Klebsiella pneumoniae (74 \%) was the most prevalent ESBL producer. This result was concordant with studies conducted by Shivprakasha et al $(67.4 \%)^{[18]}$, and Mathur et al $(68 \%)^{[15]}$.

Studies conducted by Manchanda et al ${ }^{[20]} \&$ Nevine et al ${ }^{[7]}$ have shown a much higher prevalence of $87 \%$ \& $88.5 \%$ respectively. High percentage of ESBL producing Klebsiella pneumoniae in our study may be due to the selective pressure imposed by extensive use of antimicrobials. Patient to patient transfer of ESBL producing strains of Klebsiella spp. could also be another reason as has been shown by Jain et al ${ }^{[21]}$ in their study. In our study the prevalence of E. coli was observed to be $62 \%$ which was in accordance with studies conducted by Shivprakasha et al $(62.34 \%)^{[18]}$, and Gupta et al $(63.79 \%)^{[19]}$. In our study Klebsiella pneumoniae accounted for the most prevalent ESBL producing organism in all clinical samples which was concordant with studies of Mathur et al $(68 \%)^{[15]}$, Gupta et al $(71 \%)^{[19]}$ and Nevine et al $(88.5 \%)^{[7]}$. High prevalence of ESBL positivity in Klebsiella isolates was seen in samples of Blood (86\%), Urine $(86 \%)$ and fluid samples $(82 \%)$, similar results were obtained by Gupta et $\mathrm{al}^{\left[{ }^{[19]}\right.}$ also.

After screening all the GNBs according to CLSI recommended screening criteria, 519 (73\%) were found to be potential ESBL producers. However, ESBL production could be confirmed in only $433(83.4 \%)$ by the phenotypic confirmatory method. Thus in our study the confirmatory method could not detect ESBL production in 86 isolates $(16.5 \%)$. Such discrepancy between screening and confirmation test results has also been observed in other studies ${ }^{[9][22]}$. This discrepancy in test results may be because of additional resistance mechanisms, which give false negative results. These include AmpC enzymes, porin changes and TEM and SHV $\beta$-lactamases with decreased affinities for $\beta$-lactamase inhibitors that can mask clavulanic acid inhibition giving false negative test results ${ }^{[22]}$.

In our study we observed that co-resistance to non $\beta$-lactam antibiotics was more with ESBLs $(\mathrm{P}<$ 0.0001), which was highly significant. This was in accordance with other studies done by, Shivaprakasha et al ${ }^{[18]}$, Jain et al ${ }^{[21]}$ and Menon et al ${ }^{[13]}$. The resistance conferred by ESBLs in our study for Amikacin, Ciprofloxacin and Gentamicin was found to be $46 \%, 59 \%$ and $63 \%$ respectively. According to our study Imipenem was found to be the most effective drug against ESBL producers $(97 \%)$ as was seen in other studies $[13][14]$

\section{Conclusion}

Detection and reporting of $\beta$-lactamase producers is the responsibility of every clinical Microbiologist. Each laboratory should have one interested, expert staff solely dedicated to follow up important observation with respect to development of new resistant mechanisms and method to detect them till CLSI recommended tests becomes available. Because bacterial pathogens are constantly changing, training must be an ongoing process. To prevent the spread of ESBL producing organism, infection control precautions like barrier nursing, cohorting of patients and nurses, attention to hand washing are essential. Development of an infection control policy and hospital antibiotic prescribing guide should be followed. Education of medical and nursing staffs, patients, visitors and medical students through handouts, posters and meetings could play an important part. 
Knowledge of the resistance pattern of bacterial strains in a geographical area will help to guide the appropriate and judicious antibiotic use. Empirical use of antibiotics can give rise to a new dawn of decreased resistant bacterial era.

\section{Reference}

[1]. Nurul M A, Loo H K C, Subramaniam G, Wong E H, Selvi P, Ho S, Kamarulzaman A. and Parasakthi N. Faecal prevalence of extended-spectrum $\beta$ - lactamase (ESBL)-producing coliforms in a geriatric population and among haematology patients. Malaysian J Pathol 2005; 27(2): $75-81$.

[2]. Ali A M, Rafi S, Qureshi AH. Frequency of Extended Spectrum Beta Lactamase Producing Gram Negative Bacilli among Clinical Isolates at Clinical Laboratories of Army Medical College, Rawalpindi. http://ayubmed.edu.pk/JAMC/PAST/16-1/Aarif.htm

[3]. David L. Paterson and Robert A. Bonomo. Extended-Spectrum B-Lactamases: A Clinical Update. Clinical Microbiology Reviews 2005: 18(4): 657-686.

[4]. Sanders CC, Barry AL, Washington JA, Shubert C, Moland ES, Traczewski MM, Knapp C, Mulder R. Detection of ESBL producing members of the family Enterobacteriaceae with the Vitek ESBL test. J Clin Microbiol 1996; 34: $2997-3001$.

[5]. Andrea J. Linscott and William J. Brown. Evaluation of Four Commercially Available Extended-Spectrum Beta-Lactamase Phenotypic Confirmation Tests. Journal of Clinical Microbiology 2005; 43(3): 1081-1085.

[6]. Chaudhary U, Aggarwal R. Extended spectrum -lactamases (ESBL) - An emerging threat to clinical therapeutics. Indian Journal of Medical Microbiology 2004; 22: 75-80.

[7]. Nevine S. Fam, M M. El-Damarawy. CTX-M-15 Extended-Spectrum Beta-Lactamases Detected from Intensive Care Unit of an Egyptian Medical Research Institute. Research Journal of Medicine and Medical Sciences 2008; 3(1): 84-91.

[8]. Hyunjoo Pai. Clinical Implications of ESBLs. Lectures ISAAR 2003. http://www.ansorp.org/ansorp2008/isaar/down/2003/sb_ Hyunjoo_Pai.pdf

[9]. Shobha K.L, Ramachandra L, Rao G, Majumder S, Rao S P. Extended Spectrum Beta-Lactamases (ESBL) In Gram Negative Bacilli At A Tertiary Care Hospital. Journal of Diagnostic and Clinical Research 2009; 3(1): 1307-1312.

[10]. Colle J G, Miles B P, Wan B. Tests For The Identification Of Bacteria. In: Collee J G, Fraser A G, Marmion B P, Simmons A, editors. Mackie and McCartney Practical Medical Microbiology, $14^{\text {th }}$ edition. Edinburg; Churchill Livingstone; 1996 : $131-150$.

[11]. Bauer AW, Kirby WMM, Sherris JC, Tuck M. Antibiotic Susceptibility Testing By Standardized Single Disc Method. Am J Clin Pathol 1966; 45: 493-6.

[12]. Clinical Laboratory Standard Institute. Performance standards for antimicrobial susceptibility testing. 17th Informational Supplement. Wayne, Pennsylvania: CLSI 2007; M100-S17: 27(3).

[13]. Menon T, Bindu D, Kumar CPG, Nalini S, Thirunarayan MA. Comparison of Double Disc and Three Dimensional Methods to Screen for ESBL Producers in a Tertiary Care Hospital. Indian Journal of Medical Microbiology 2006; 24 (2):117-120.

[14]. Babypadmini S, Appalaraju B. Extended Spectrum Beta Lactamases In Urinary Isolates Of E. coli \& K. pneumoniae-Prevalence And Susceptibility Pattern In A Tertiary Care Hospital. Indian J Med Microbiol 2004; 22: 172-4.

[15]. Mathur P, Kapil A, Das B, Dhawan B. Prevalence of extended spectrum beta lactamase producing Gram negative bacteria in a tertiary care hospital. Indian J Med Res 2002; 115: 153-7.

[16]. Rodrigues C, Joshi P, Jani S H, Alphonse M, Radhakrishnan R, Mehta A. Detection Of $\beta$-Lactamases In Nosocomial Gram Negative Clinical Isolates. Indian Journal of Medical Microbiology 2004; 22(4):247-250

[17]. Khurana S, Taneja N, Sharma M. Extended Spectrum $\beta$-Lactamase Mediated Resistance In Urinary Tract Isolates Of Family Enterobacteriaceae. Indian J Med Res 2002; 116:145-149.

[18]. Shivaprakasha S., Radhakrishnan K., Gireesh A. \& Shamsul Karim P.: Routine Screening for ESBL Production, A Necessity Of Today. The Internet Journal of Microbiology. 2007; 3 (1).

[19]. Gupta V, Singla N \& Jagdish C. Detection Of Sells Using Third \& Fourth Generation Cephalosporins In Double Disc Synergy Test. Indian J Med Res 2007; 126: 486-487.

[20]. Manchanda V, Singh NP, Goyal R, Kumar A, Thukral SS. Phenotypic Characteristics Of Clinical Isolates Of Klebsiella pneumoniae And Evaluation Of Available Techniques For Detection Of Extended Spectrum Beta-Lactamases. Indian J Med Res 2005; 122: 330-7.

[21]. Jain A, Roy I, Gupta K, Kumar M and Agarwal S.K. Prevalence of ESBL Producing Gram Negative Bacteria in Septicaemic Neonates in Tertiary Care Hospital. J Medical Microbiology 2003; 52: 421-425.

[22]. Sanguinetti, M., B. Posteraro, T. Spanu, D. Ciccaglione, L. Romano, B. Fiori, G. Nicoletti, S. Zanetti, and G. Fadda. Characterization of clinical isolates of Enterobacteriaceae from Italy by the BD Phoenix extended-spectrum beta-lactamase detection method. J. Clin. Microbiol 2003; 41: 1463-1468. 